\title{
A NONCOMMUTATIVE EXTENSION OF THE PERRON-FROBENIUS THEOREM
}

\author{
BY LEONARD GROSS ${ }^{1}$
}

\author{
Communicated by R. A. Kunze, November 18, 1970
}

\begin{abstract}
The existence and uniqueness of physical ground states is proved for various interactions in quantum field theory using some infinite dimensional extensions of the Perron-Frobenius theorem.
\end{abstract}

Let $a$ be a von Neumann algebra with a finite regular trace. A bounded linear operator $A$ from $L^{2}(Q)$ to $L^{2}(Q)$ is of nonnegative type if $A$ takes nonnegative operators to nonnegative operators. The classical theorem of Perron and Frobenius [2] asserts that if $a$ is finite dimensional (in which case $L^{2}(Q)=Q$ ) and commutative and if $A$ is of nonnegative type and has spectral radius $r$ then $r$ is an eigenvalue of $A$ and has a corresponding eigenvector which is nonnegative. If, in addition, $A$ leaves no proper ideals of $a$ invariant then $r$ is an algebraically simple eigenvalue.

In this note we remove the assumptions that $Q$ is finite dimensional and commutative. Denoting by $L_{\alpha}$ and $R_{\alpha}$ the bounded operators of left and right multiplication on $L^{2}(Q)$ by an element $\alpha$ in the von Neumann algebra $Q$ we put, for any projection $e$ in $Q, P_{e}=L_{e} R_{e} . P_{e}$ is a projection on $L^{2}(Q)$. Its range will be called a Pierce subspace. We consider a bounded Hermitian operator $A$ of nonnegative type on $L^{2}(Q)$. We shall show that if, for some $p>2, A$ is bounded from $L^{2}(Q)$ to $L^{p}(Q)$ then $r=\|A\|$ is an eigenvalue of $A$ and $A$ has a nonnegative eigenvector with eigenvalue $r$. If, in addition, $A$ leaves invariant no proper Pierce subspace then $r$ has multiplicity one.

This work is motivated by our attempts to prove the existence and uniqueness of physical ground states in various models in quantum field theory. Among new results following from these methods is existence of the physical one particle rest state for some interactions involving Bosons, where $Q$ is commutative, and uniqueness of the physical vacuum for interactions involving Fermions, where $Q$ is a

AMS 1970 subject classifications. Primary 15A48, 15A66, 47A55, 81A18; Secondary 46L10, 47B55, 81A17.

Key words and phrases. Perron-Frobenius theorem, existence of physical ground states, uniqueness of physical ground states, Fermions, Bosons, von Neumann algebras.

1 Research partially sponsored by the U. S. Air Force Office of Scientific Research, Office of Aerospace Research under Grant No. AF-AFOSR 743-67. 
Clifford algebra. Various known results concerning the existence of the physical vacuum also follow from these methods. [8].

For the relevant noncommutative integration theory see [6], [7],

Theorem 1 (EXISTENCE). Let $(\Omega, \mu)$ be a finite measure space. Let $A$ be a bounded Hermitian operator on $L^{2}(\Omega)$ of nonnegative type. Put $r=\|A\|$. If for some real number $p>2$ there is a real constant $M$ such that $\|A \psi\|_{L^{p}} \leqq M\|\psi\|_{L^{2}}$ then $r$ is an eigenvalue of $A$ with finite multiplicity. A has nonnegative eigenvectors with eigenvalue $r$.

TheOREM 2 (EXISTENCE). Let a be a von Neumann algebra with a finite regular trace. Assume that a contains a directed system of finite dimensional * subalgebras with union dense in $L^{2}(Q)$. Let $A$ be a bounded Hermitian operator on $L^{2}(Q)$ of nonnegative type. Put $r=\|A\|$. If for some real number $p>2$ there is a real constant $M$ such that $\|A \psi\|_{p} \leqq M\|\psi\|_{2}$ then $r$ is an eigenvalue of $A$. A has nonnegative eigenvectors with eigenvalue $r$.

To prove Theorem 1 let $\alpha=\left\{E_{1}, \cdots, E_{n}\right\}$ be a partition of $\Omega$ into measurable sets of positive measure. Let $P_{\alpha}$ be the corresponding conditional expectation projection on $L^{2}(\Omega)$. Then $P_{\alpha} A P_{\alpha}$ is of finite rank and of nonnegative type and hence has a nonnegative unit eigenvector $\psi_{\alpha}$ of eigenvalue $\left\|P_{\alpha} A P_{\alpha}\right\|$. One shows that weak $\lim _{\alpha} \psi_{\alpha}$ is not zero when the collection of partitions is directed by refinement.

Theorem 3 (Uniqueness of MAXIMUM EIGenVector). Let $a$ be $a$ von Neumann algebra with a finite regular trace. Let $A$ be a bounded Hermitian operator on $L^{2}(Q)$ of nonnegative type. Assume that $r=\|A\|$ is an eigenvalue of $A$. If $A$ leaves invariant no proper Pierce subspace of $L^{2}(Q)$ then $r$ has multiplicity one.

Remark. The algebraic hypothesis in this theorem, that $A$ leave invariant no proper Pierce subspace, is equivalent to an analytic condition, ergodicity [4], which is a notion that can be formulated in a much more general context [5] than that with which we are dealing. The virtue of this algebraic hypothesis lies in the fact that it is very easy to verify it for a large class of operators that arise in quantum field theory. The first assertion in the following perturbation theorem illustrates this.

THEOREM 4. Let a be a von Neumann algebra with a finite regular trace. Let $H_{0}$ be a selfadjoint operator on $L^{2}(Q)$ such that $H_{0} \geqq 0$. Assume that the null space of $H_{0}$ is spanned by the identity element of $Q$ and let a be a Hermitian element in a. Put $B=L_{a}+R_{a}$ and $H=H_{0}+B$. If $t$ $>0$ then $\exp (-t H)$ leaves no proper Pierce subspace invariant. If 
$\exp \left(-t H_{0}\right)$ is of nonnegative type for all $t>0$ then so is $\exp (-t H)$. If $\exp \left(-t H_{0}\right)$ is bounded from $L^{2}(Q)$ to $L^{p}(Q)$ for some $p>2$ and some $t>0$ then $\exp (-t H)$ is bounded from $L^{2}(Q)$ to $L^{p}(Q)$ for some $p>2$ and some $t>0$.

We prove the first assertion of the theorem. Suppose $e$ is a nonzero projection in $Q$ such that $P_{e}$ commutes with $\exp (-t H)$ for some $t>0$. Since $H$ is selfadjoint, $P_{e}$ commutes with $\exp (-t H)$ for all $t \geqq 0$. Now 1 is in the domain of $H$. Hence both sides of the equation

$$
P_{e} \exp (-t H) 1=\exp (-t H) P_{e} 1
$$

are differentiable at $t=0$. Consequently $e=P_{e} 1$ is in the domain of $H$ and

$$
P_{e}(H 1)=H e \text {. }
$$

Now $P_{e} H 1=P_{e}(2 a)=2 e a e$ and $H e=H_{0} e+a e+e a$. Thus $2 e a e=H_{0} e$ $+a e+e a$. Apply $P_{e}$ to obtain $P_{e}\left(H_{0} e\right)=0$. Taking the inner product with 1 we get $0=\left(P_{\theta} H_{0} e, 1\right)=\left(H_{0} e, e\right)$. Since $H_{0} \geqq 0$ it follows that $H_{0} e=0$. Hence $e=1$ and $P_{e}$ is the identity operator.

REMARK. The conclusions of Theorem 4 remain true under a considerable relaxation of the requirement that $a$ be in $Q$. Specifically, noncommutative extensions of the Stein interpolation theorem combined with techniques of Segal [10], [11] allow one to extend Theorem 4 to a large class of perturbations of the form $B=L_{a}+R_{a}$ where $a$ is unbounded. But the part of the proof given above remains unchanged if $H_{0}+B$ is selfadjoint on its domain (and bounded below).

Applications. Let $\mathfrak{H C}$ be a complex Hilbert space and let $J$ be a conjugation on $\mathcal{H C}$. Denote by $\Lambda^{n}(\mathcal{H C})$ the space of skew symmetric $n$ tensors over $\mathfrak{F C}$ with $\Lambda^{0}(\mathcal{H C})=$ complex numbers. Put $\Lambda(\mathcal{H C})=\sum_{n=0}^{\infty} \Lambda^{n}(\mathfrak{F C})$ and $\psi_{0}=1$ in $\Lambda^{0}(\mathcal{F C}) \subset \Lambda(\mathcal{F C})$. For each $x$ in $\mathfrak{F C}$ there is a unique bounded operator $C_{x}$ on $\Lambda(\mathcal{H C})$ such that $C_{x} u=(n+1)^{1 / 2} x \wedge u$ when $u \in \Lambda^{n}(\mathcal{H C})$. Let $A_{x}=C_{x}^{*}$ and put $L_{x}=C_{x}+A_{J x}$. Let $\mathcal{C}$ be the von Neumann algebra generated by the operators $\left\{L_{x}: x \in \mathfrak{F}\right\}$. Then $\mathcal{C}$ is a factor of type $\mathrm{II}_{1}$ and trace $(a)=\left(a \psi_{0}, \psi_{0}\right)$ is a finite regular trace on $\mathcal{C}$. $\mathcal{C}$ is the weakly closed Clifford algebra over $\mathfrak{H C}$ relative to the conjugation $J$. $\psi_{0}$ is a separating cyclic vector for $\mathcal{C}$ and the map $a \rightarrow a \psi_{0}$ extends to a unitary map of $L^{2}(\mathcal{C})$ onto $\Lambda(\mathcal{H C})$, cf. [9]. Let $D$ be a selfadjoint operator on $\mathfrak{F C}$ such that $D \geqq m I$ for some constant $m>0$. The one parameter unitary group $\exp (i t D)$ extends to a one parameter unitary group on $\Lambda(\mathcal{H C})$ whose infinitesimal generator we denote by $H_{1}$. In view of the isomorphism of $L^{2}(\mathcal{C})$ with $\Lambda(\mathcal{H C})$ we may regard $H_{1}$ as an operator on $L^{2}(\mathcal{C})$. If $D$ commutes with $J$ then $H_{1} \geqq 0, \exp \left(-t H_{1}\right)$ is of nonnegative type for each $t>0$, and for each $t>0$ there is a $p>2$ 
such that $\exp \left(-t H_{1}\right)$ is bounded from $L^{2}(\mathcal{C})$ to $L^{p}(\mathfrak{C})$. The null space of $H_{1}$ is spanned by the identity of $\mathfrak{e}$. Thus if $a$ is in $\mathfrak{e}$ and $H=H_{1}$ $+L_{a}+R_{a}$ then, by Theorems 2,3 , and $4, H$ has a simple eigenvalue at the lower end of its spectrum.

It is remarkable that the total Hamiltonian for a Fermion field interacting with an external scalar field via a cutoff Yukawa interaction has the form $H=H_{1}+L_{a}+R_{a}$ described above when the conjugation $J$ on the one particle nucleon-anti-nucleon space $\mathfrak{H C}$ is suitably chosen. A suitable choice for $J$ in four space-time dimensions is $J=T C P$ restricted to $\mathcal{H C}$ where $T$ is Wigner time reversal, $C$ is charge conjugation and $P$ is intrinsic parity (not space reflection). See $[1$, pp. 109, 112] for a discussion of intrinsic parity. Thus the existence and uniqueness of the physical vacuum for this model follows from the methods described in this note. We mention also that the terms $L_{a}+R_{a}$ in the total Hamiltonian correspond to the pair creation and annihilation terms of the interaction Hamiltonian and that $H_{1}$ includes the free Hamiltonian plus the other terms of the interaction Hamiltonian plus a mass renormalization term.

As a second application we derive the existence of physical one particle rest states for a massive polaron model from Theorem 1 . The uniqueness follows from the mentioned extension of Theorem 4 . We let $\Re=\operatorname{real} L^{2}\left(R^{n}\right)$. Let $h$ be a fixed real continuous function in $L^{2}\left(R^{n}\right)$. For each $x$ in $R^{n}$ let $F_{x}(\varphi)=(\varphi * h)(x)$ where $*$ denotes convolution and $\varphi$ is in $\nVdash$. Then $F_{x}$ is a real continuous linear functional on $\nVdash$ for each $x$. Let $(\Omega, \nu)$ be a probability space associated with the isotropic normal distribution on $\mathscr{K}$ with variance parameter one. Then, for each $x$ in $R^{n}, F_{x}$ corresponds to a measurable function $\varphi_{h}(x)(\cdot)$ on $\Omega$. Multiplication by $\varphi_{h}(x)(\cdot)$ on $L^{2}(\Omega)$ may be regarded as a Boson field at time zero with cutoff function $h$. The Hilbert space for a massive polaron at rest may be taken to be $L^{2}(\Omega)$. The total Hamiltonian with cutoff $h$ may then be described thus: Let $T_{x}$ denote space translation in $\mathscr{K},\left(T_{x} \psi\right)(y)=\psi(y-x)$. Then $T_{x}$ is an orthogonal transformation of $\pi$ and therefore induces a unitary transformation $\hat{T}_{x}$ of $L^{2}(\Omega)$ which can profitably be thought of as being induced by a measure preserving transformation of $\Omega$. The map $x \rightarrow \hat{T}_{x}$ is a strongly continuous unitary representation of the additive group of $R^{n}$. We denote the infinitesimal generators relative to some O.N. basis of $R^{n}$ by $P_{1}, \cdots, P_{n}$. Thus $\hat{T}_{x}=\exp \left(i \sum_{j=1}^{n} x_{j} P_{j}\right)$. Let $E(p), p$ in $R^{n}$, be the kinetic energy function of the nucleon. The total Hamiltonian is then $H=E\left(\left(P_{1}, \cdots, P_{n}\right)\right)+\left(H_{0}\right)_{\text {Boson }}+$ multiplication by $\varphi_{h}(0)$. It is well understood [4], [10], [11] why the contribution to $\exp (-t H)$ from the last two terms yields an operator of nonnegative type on $L^{2}(\Omega)$. We limit our discussion to $\exp \left(-t E\left(P_{1}, \cdots, P_{n}\right)\right)$. If 


$$
\exp (-t E(p))=\int_{R^{n}} g_{t}(x) \exp (i x \cdot p) d x
$$

then

$$
\begin{aligned}
\exp \left(-t E\left(\left(P_{1}, \cdots, P_{n}\right)\right)\right) & =\int_{R^{n}} g_{t}(x) \exp \left(i \sum_{j=1}^{n} x_{j} P_{j}\right) d x . \\
& =\int_{R^{n}} g_{t}(x) \hat{T}_{x} d x .
\end{aligned}
$$

Now $\hat{T}_{x}$ is of nonnegative type since it is (essentially) induced by a measure preserving transformation of $\Omega$. Hence if $g_{l}(x) \geqq 0$ for all $t>0$ and all $x$ then $\exp \left(-t E\left(\left(P_{1}, \cdots, P_{n}\right)\right)\right)$ will be of nonnegative type. Thus a sufficient condition for the nonnegativity of $\exp (-t H)$ is that $\exp (-t E(p))$ be a positive definite function of $p$ for all $t>0$. This condition is satisfied in both the Newtonian case where $E(p)$ $=|p|^{2} / 2 m$, and the relativistic case where $E(p)=\left(m^{2}+|p|^{2}\right)^{1 / 2}$. The requirement in Theorem 1 that $\exp (-t H)$ be bounded from $L^{2}$ to $L^{p}$ for some $p>2$ is easily established using known techniques.

\section{REFERENCES}

1. J. D. Bjorken and S. D. Drell, Relativistic quantum fields, McGraw-Hill, New York, 1965. MR 32 \#5092.

2. G. Frobenius, Über Matrizen aus positiven Elementen, S.-B. Preuss. Akad. Wiss., Berlin 1908, 471-476; O. Perron, Zur Theorie der Matrices, Math. Ann. 64 (1907), 248-263.

3. F. R. Gantmacher, Applications of the theory of matrices, GITTL, Moscow, 1953; English transl., Interscience, New York, 1959. MR 16, 438; MR 21 \#6372b.

4. J. Glimm and A. Jaffe, $A \lambda\left(\varphi^{4}\right)_{2}$ quantum field theory without cutoffs. II, Ann. of Math. (2) 91 (1970), 362-401.

5. M. G. KreYn and M. A. Rutman, Linear operators leaving invariant a cone in a Banach space, Uspehi Mat. Nauk 3 (1948), no. 1 (23), 3-95; English transl., Amer. Math. Soc. Transl. (1) 10 (1962), 199-325. MR 10, 256; MR 12, 341.

6. R. A. Kunze, $L_{p}$ Fourier transforms on locally compact unimodular groups, Trans. Amer. Math. Soc. 89 (1958), 519-540. MR 20 \#6668.

7. I. E. Segal, A non-commutative extension of abstract integration, Ann. of Math.

(2) 57 (1953), 401-457. MR 14, 991.

8. - Correction to "A non-commutative extension of abstract integration," Ann. of Math. (2) 58 (1953), 595-596. MR 15, 204.

9. - Tensor algebras over Hilbert spaces. II, Ann. of Math. (2) 63 (1956), 160-175. MR 17, 1114.

10. - Notes towards the construction of nonlinear relativistic quantum fields. III. Properties of the $C^{*}$-dynamics for a certain class of interactions, Bull. Amer. Math. Soc. 75 (1969), 1390-1395. MR 40 \#5217.

11. - Construction of non-linear local quantum processes. I, Ann. of Math. (2) 92 (1970), 462-481.

Cornell University, Ithaca, New York 14850 